\title{
Association between Intrapancreatic Fat Deposition and the Leptin/Ghrelin Ratio in the Fasted and Postprandial States
}

\author{
Ngoc N. Nguyen Ruma G. Singh Maxim S. Petrov \\ School of Medicine, University of Auckland, Auckland, New Zealand
}

\section{Keywords}

Leptin · Ghrelin · Intrapancreatic fat · Energy balance

\begin{abstract}
Background: The clinical relevance of excess intrapancreatic fat deposition (IPFD) is increasingly appreciated. Leptin and ghrelin are key players in the regulation of food intake, energy balance, and body fat mass. The aim was to investigate the associations of the leptin/ghrelin ratio and its components with IPFD. Methods: All participants underwent magnetic resonance imaging on a $3 \mathrm{~T}$ scanner to quantify IPFD. Both fasting and postprandial blood samples were analyzed for leptin and acylated ghrelin. Linear regression analysis was conducted, accounting for visceral/subcutaneous fat volume ratio, glycated hemoglobin, and other covariates. Results: A total of 94 participants ( 32 women) with a median age of 56 (interquartile range 44-66) years were studied. Their median IPFD was 9.6\% (interquartile range 8.8-10.4\%). In the fasted state, the leptin/ghrelin ratio $(\beta=0.354 ; 95 \%$ confidence interval $0.044-0.663 ; p=0.025$, in the most adjusted model) and leptin ( $\beta=0.040 ; 95 \%$ confidence interval $1.003-1.078 ; p=0.035$, in the most adjusted model) were significantly associated with IPFD. Ghrelin in the fasted state was not significantly associated with IPFD. In the postpran-
\end{abstract}

karger@karger.com www.karger.com/anm

Karger $\stackrel{\text { ' }}{5}$
(C) 2021 The Author(s)

Published by S. Karger AG, Basel

This article is licensed under the Creative Commons Attribution 4.0 International License (CC BY) (http://www.karger.com/Services/ OpenAccessLicense). Usage, derivative works and distribution are permitted provided that proper credit is given to the author and the original publisher. dial state, the leptin/ghrelin ratio, leptin, and ghrelin were not significantly associated with IPFD. Conclusion: Fasting circulating levels of leptin are directly associated with IPFD. Purposely designed mechanistic studies are warranted to determine how high leptin may contribute to excess IPFD.

(c) 2021 The Author(s).

Published by S. Karger AG, Basel

\section{Introduction}

Fatty pancreas disease is the most common pathology of the pancreas, with the prevalence of at least $16 \%$ in the general population $[1,2]$. It is associated with a 1.7 -times increased risk of arterial hypertension, 2.1-times increased risk of diabetes, and 2.4-times increased risk of metabolic syndrome [3]. It also affects considerably diseases of the exocrine pancreas, such as pancreatitis and pancreatic cancer [4-6]. Importantly, fatty pancreas disease is a potentially reversible pathology as intrapancreatic fat deposition (IPFD) can be significantly reduced in response to nutritional interventions. Intermittent fasting, as compared with high-fat diet, was shown to significantly reduce IPFD [7]. Reduced IPFD following a lowcalorie diet ( $700 \mathrm{kcal} /$ day) in individuals with diabetes led to normalization of blood glucose [8]. Moreover, only 
people with remission of diabetes (but not those who had diabetes at 24 months of follow-up) had a significantly reduced IPFD [9]. Also, a randomized controlled trial of Mediterranean diet (low in carbohydrates and rich in unsaturated fats) versus low-fat diet (both diets aimed at an energy intake of 1,500-1,800 kcal/day) demonstrated that the former results in a significantly lower IPFD [10].

The pathophysiological mechanisms underlying fatty pancreas disease are yet to be fully elucidated. The reduction in IPFD following certain diets points to the possible role of the energy balance and food intake, known to be regulated in antagonistic fashion by 2 hormones - leptin and ghrelin. The "satiety hormone" leptin is a 167-aminoacid peptide predominantly secreted by adipocytes. It regulates food intake mainly by stimulating anorexigenic (pro-opiomelanocortin) neurons and inhibiting orexigenic (neuropeptide $\mathrm{Y}$ and Agouti-related protein) neurons in the arcuate nucleus of the hypothalamus [11]. Most of the effects of leptin on end organs and systems are indirect via the central nervous system. The "hunger hormone" ghrelin is a 28 -amino-acid peptide predominantly secreted by enterochromaffin cells located in the stomach. The orexigenic effect of ghrelin is mediated via central activation of neuropeptide $\mathrm{Y}$ pathways, which causes a short-term increase in food intake. It also stimulates appetite by increasing gastrointestinal motility and reducing the secretion of insulin [12]. Leptin and ghrelin are reciprocally regulated, and the usefulness of studying the ratio of their concentrations (originally called the "ghrelin-leptin tango" [13]) has been demonstrated in people with obesity. However, a systematic literature review by the COSMOS group has found no study to investigate the leptin/ghrelin ratio specifically in the context of IPFD and taking into account visceral and subcutaneous fat volumes [14]. The aim of the present study was to investigate the associations between IPFD and the leptin/ ghrelin ratio, leptin, and ghrelin in both fasted and postprandial states.

\section{Methods}

\section{Study Design and the Eligibility Criteria}

This was a cross-sectional study as part of the ARIES project [15]. The study was conducted at the COSMOS clinic (University of Auckland) and the university's center of advanced magnetic resonance imaging (MRI). Participants were included if they were at least 18 years of age, provided informed consent, resided in Auckland at the time of the study, and had a history of acute pancreatitis (no earlier than 3 months prior to the study date). Diagnosis of acute pancreatitis required the presence of at least 2 of the following 3 criteria: characteristic findings on computed tomography,
MRI, or ultrasonography during hospitalization; pain typical of acute pancreatitis; and serum amylase and/or lipase levels that are $>3$ times higher the upper limit of normal. Participants were excluded if they had post-endoscopic retrograde cholangiopancreatography pancreatitis, chronic pancreatitis, hereditary pancreatitis, pancreatic cancer (or another malignancy), pancreatic lipomatosis or lipomatous pseudohypertrophy, congenital anomalies of the pancreas, cognitive disability, and cystic fibrosis, received surgical, endoscopic, or radiologic interventions involving the pancreas, received steroid therapy, were pregnant or postpartum, and had metallic foreign body implantations, heart pacemakers, or other implanted electronic devices.

\section{Quantification of Fat Depots}

All study participants underwent abdominal MRI on a 3.0 Tesla MAGNETOM Skyra MRI Scanner (Siemens, Erlangen, Germany), specifically for the purpose of the ARIES project. The MRI acquisition parameters were detailed elsewhere [16]. The technique used to quantify IPFD was described in detail elsewhere [17]. In brief, 3 regions of interest were placed in the head, body, and tail regions of the pancreas of 2 candidate slices. A thresholding range of $1-20 \%$ was applied to avoid nonparenchymal tissue within the selected regions of interest. The average measurement of the 2 candidate slices was used for statistical analyses. The ratio of visceral-to-subcutaneous (V/S) fat volume was also calculated as described elsewhere [18]. Two raters, blinded to the participant groups, independently carried out the measurements of IPFD, subcutaneous fat volume, and visceral fat volume. The inter-rater reliability of the MRI measurements was determined by intraclass correlation coefficients [19]. Statistical analyses used the average values of 2 independent MRI measurements for each fat depot.

\section{Measurements of Leptin and Ghrelin}

All study participants fasted for at least $8 \mathrm{~h}$ before blood collection. These blood samples were used for the fasted state analyses. A subset of participants also had serial blood samples (at 15, 30, 45, 60,90 , and $120 \mathrm{~min}$ ) collected following a standardized mixedmeal test (BOOST drink providing 360 calories, 15 g protein, 61.5 $\mathrm{g}$ carbohydrate, and $6 \mathrm{~g}$ fat), described in detail elsewhere [20]. Serine protease inhibitor was added to blood samples for acylated ghrelin (i.e., peptide containing the octanoyl group, which is essential for binding to the ghrelin receptor) measurement. All blood samples were centrifuged at $4,000 \mathrm{~g}$ for $5 \mathrm{~min}$, serum separated into aliquots, and stored at $-80^{\circ} \mathrm{C}$ for further use. Levels of leptin and ghrelin were measured using MILLIPLEX ${ }^{\circledR}$ MAP (Merck KGaA, Darmstadt, Germany) in line with the user's manual. Both leptin and ghrelin had the intra-assay and inter-assay coefficients of variation of $<10 \%$ and $<15 \%$, respectively.

\section{Statistical Analysis}

Statistical analysis was conducted using IBM SPSS Statistics Mac Version 25 (SPSS Inc., Chicago, IL, USA). $\chi^{2}$ test and independent $t$ test were used to compare baseline characteristics of the overall cohort and the subset of participants who underwent the mixed-meal test. Categorical and continuous variables were presented as frequency (percentage) or median (interquartile range), respectively. Linear regression analysis using generalized linear models of data in the fasted state was performed to investigate the associations between IPFD and the leptin/ghrelin ratio, leptin, 
Table 1. Study characteristics

\begin{tabular}{|c|c|c|c|}
\hline Characteristics & Fasted state $(n=94)$ & Postprandial state $(n=37)$ & $p$ value \\
\hline Age, years & $56(44-66)$ & $56(41-66)$ & 0.941 \\
\hline Sex & & & 0.684 \\
\hline Men & $62(65.9)$ & $26(70.3)$ & \\
\hline Women & $32(34.1)$ & $11(29.7)$ & \\
\hline Weight, kg & $84(72-99)$ & $86(73-102)$ & 0.878 \\
\hline Height, m & $1.72(1.65-1.81)$ & $1.75(1.66-1.84)$ & 0.759 \\
\hline Body mass index, $\mathrm{kg} / \mathrm{m}^{2}$ & $27.5(24.5-33.4)$ & $27.8(24.9-33.8)$ & 0.802 \\
\hline Visceral fat volume, $\mathrm{L}$ & $1.94(1.23-2.67)$ & $1.86(1.13-2.47)$ & 0.610 \\
\hline Subcutaneous fat volume, $\mathrm{L}$ & $2.88(2.09-4.08)$ & $2.74(2.03-3.84)$ & 0.483 \\
\hline Glycated hemoglobin, $\mathrm{mmol} / \mathrm{mol}$ & $37(34-41)$ & $38(35-40)$ & 0.413 \\
\hline Total cholesterol at baseline, $\mathrm{mmol} / \mathrm{L}$ & $5.0(4.0-5.7)$ & $4.8(3.9-5.5)$ & 0.845 \\
\hline $\mathrm{HDL}$ cholesterol at baseline, $\mathrm{mmol} / \mathrm{L}$ & $1.3(1.1-1.6)$ & $1.2(1.0-1.6)$ & 0.715 \\
\hline LDL cholesterol at baseline, $\mathrm{mmol} / \mathrm{L}$ & $2.8(1.9-3.2)$ & $2.9(2.0-3.2)$ & 0.929 \\
\hline Triglycerides at baseline, $\mathrm{mmol} / \mathrm{L}$ & $1.5(0.9-2.5)$ & $1.6(1.0-2.5)$ & 0.821 \\
\hline Etiology & & & 0.561 \\
\hline Biliary & $44(46.8)$ & $20(54.1)$ & \\
\hline Nonbiliary & $50(53.2)$ & $17(45.9)$ & \\
\hline Recurrence & & & 0.664 \\
\hline No & $70(74.5)$ & $26(70.3)$ & \\
\hline Yes & $24(25.5)$ & $11(29.7)$ & \\
\hline APACHE II score & $5.5(3-8)$ & $7(2-10)$ & 0.385 \\
\hline Time since pancreatitis (months) & $23(15.8-43.5)$ & $19(11.5-25.5)$ & 0.190 \\
\hline
\end{tabular}

Data are presented as median (interquartile range) or frequency (percentage). APACHE, acute physiology and chronic health evaluation; HDL, high-density lipoprotein; LDL, low-density lipoprotein.

and ghrelin. Three adjusted models were built alongside the unadjusted model (model 1). Model 2 was adjusted for age and sex; model 3 - age, sex, V/S fat volume ratio, and glycated hemoglobin; and model 4 - age, sex, V/S fat volume ratio, glycated hemoglobin, etiology of pancreatitis, recurrence of pancreatitis, acute physiology and chronic health evaluation (APACHE) II score, and time since pancreatitis. All the statistical assumptions were met. Results were reported as $\beta$ coefficients, $95 \%$ confidence intervals, and $p$ values. A $p$ value of $<0.05$ was deemed to be statistically significant. Changes in postprandial levels of the leptin/ghrelin ratio, leptin, and ghrelin were analyzed by calculating the area under curve using the trapezoidal rule. Linear regression analysis was used to investigate the associations between IPFD and total areas under curve of the leptin/ghrelin ratio, leptin, and ghrelin, as described above.

\section{Results}

\section{Characteristics of Participants}

The study cohort used for the analysis in the fasted state included 94 individuals with a median age of 56 (44-66) years. Of these, there were 62 men and 32 women. A subset of 37 individuals, whose baseline characteristics did not differ significantly from the overall cohort

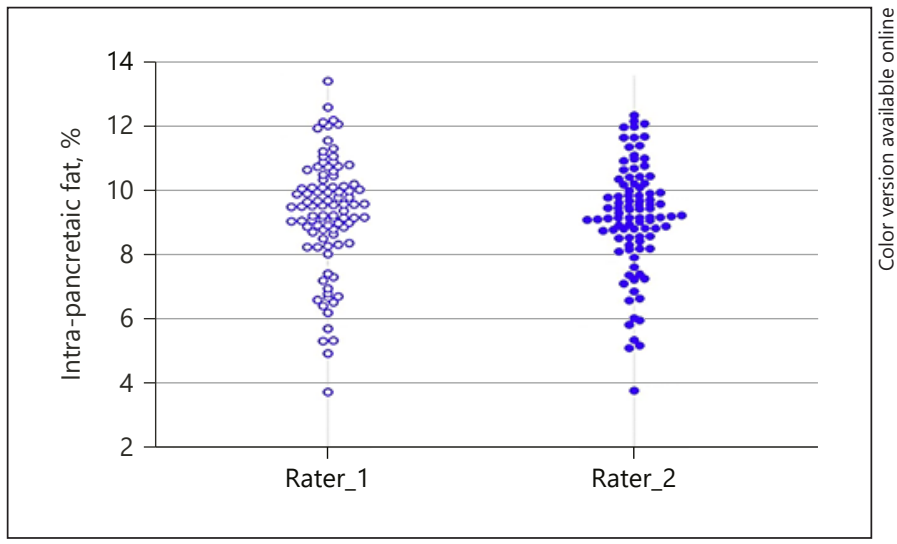

Fig. 1. Intra-class correlation plot for intrapancreatic fat measurements by 2 independent raters.

(Table 1), was used for the analysis in the postprandial state. The intra-class correlation coefficient was 0.97 for IPFD measurements by 2 independent raters (Fig. 1). Both subcutaneous fat volume and visceral fat volume measurements had the intra-class correlation coefficient of 0.99 .
16

Ann Nutr Metab 2022;78:14-20

DOI: $10.1159 / 000520068$
Nguyen/Singh/Petrov 
Table 2. The studied associations in the fasted state

\begin{tabular}{lllll}
\hline \multirow{2}{*}{ Variables } & \multirow{2}{*}{ Model } & \multicolumn{2}{l}{ Intrapancreatic fat deposition } & \\
\cline { 3 - 5 } & & $\beta$ coefficient & $95 \%$ confidence interval & $p$ value \\
\hline Leptin/ghrelin ratio & 1 & 0.266 & $-0.056,0.587$ & 0.106 \\
& 2 & 0.405 & $0.085,0.725$ & $\mathbf{0 . 0 1 3}$ \\
& 3 & 0.416 & $0.102,0.731$ & $\mathbf{0 . 0 0 9}$ \\
& 4 & 0.354 & $0.044,0.663$ & $\mathbf{0 . 0 2 5}$ \\
\hline Leptin, ng/mL & 1 & 0.034 & $-0.004,0.072$ & 0.082 \\
& 2 & 0.052 & $-0.012,0.091$ & $\mathbf{0 . 0 1 0}$ \\
& 3 & 0.046 & $0.008,0.085$ & $\mathbf{0 . 0 1 9}$ \\
& 4 & 0.040 & $0.003,0.078$ & $\mathbf{0 . 0 3 5}$ \\
\hline Ghrelin, pg/mL & 1 & 0.240 & $-0.011,0.059$ & 0.179 \\
& 2 & 0.026 & $-0.007,0.059$ & 0.129 \\
& 3 & 0.017 & $-0.017,0.050$ & 0.330 \\
& 4 & 0.028 & $-0.005,0.060$ & 0.099 \\
\hline
\end{tabular}

Model 1 was based on the univariate analysis. Model 2 was adjusted for age and sex. Model 3 was adjusted for age, sex, visceral-to-subcutaneous fat volume ratio, and glycated hemoglobin. Model 4 was adjusted for age, sex, visceral-to-subcutaneous fat volume ratio, glycated hemoglobin, acute physiology and chronic health evaluation II score, etiology, recurrence, and time since first pancreatitis episode. Significance was set at $p<0.05$. $p$ values $<0.05$ are shown in bold.

Table 3. The studied associations in the postprandial state

Associations between IPFD and the Leptin/Ghrelin

Ratio (and Its Components) in the Fasted State

IPFD was not significantly associated with the leptin/ ghrelin ratio in the univariate analysis, but was signifi- cantly associated with the ratio across all the adjusted models (Table 2). IPFD was not significantly associated with circulating levels of leptin in the univariate analysis, but was significantly associated with them across all the 

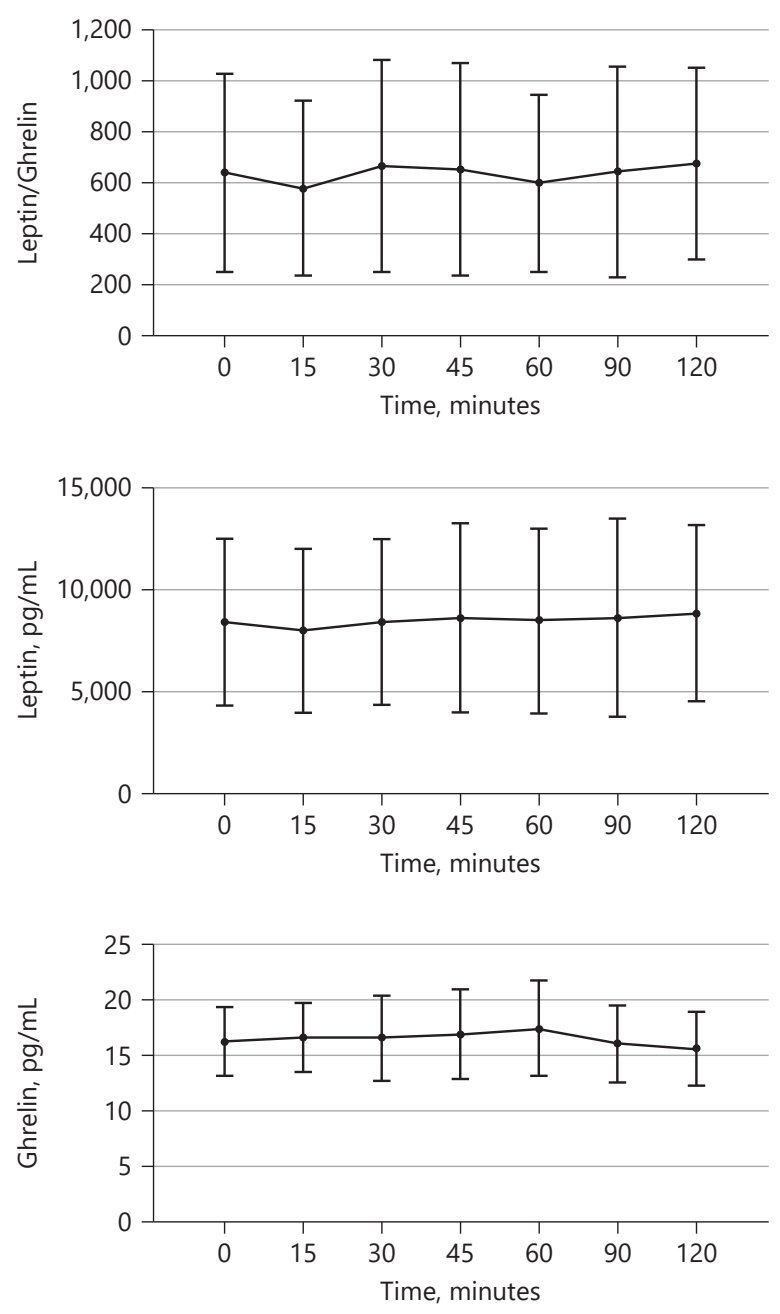

Fig. 2. Changes in the leptin/ghrelin ratio (top) and its components (middle and bottom) during the mixed-meal test. Data are presented as mean and standard error.

adjusted models (Table 2). IPFD was not significantly associated with ghrelin levels, in both the unadjusted and adjusted models (Table 2 ).

Associations between IPFD and the Leptin/Ghrelin Ratio (and Its Components) in the Postprandial State

Changes in the leptin/ghrelin ratio, leptin, and ghrelin during the mixed-meal test are presented in Figure 2. IPFD was not significantly associated with either the leptin/ghrelin ratio or ghrelin levels, in both unadjusted and adjusted models (Table 3). IPFD was significantly associated with circulating levels of leptin in the model adjusted for age and sex, but not the other models (Table 3 ).

\section{Discussion}

To the best of our knowledge, this is the first study to investigate the associations between IPFD and the leptin/ ghrelin ratio as well as its components, in both fasted and postprandial states. The main finding was that the leptin/ ghrelin ratio was significantly directly associated with IPFD in the fasted state (but not in the postprandial state). Further, this finding was driven by circulating levels of leptin, but not acylated ghrelin. Importantly, the observed associations held true independent of age, sex, abdominal fat volumes, and glycated hemoglobin - the factors that are known to affect both IPFD and the studied hormones $[3,14]$.

It is well known that, in general, circulating levels of leptin are strongly associated with fat mass in both humans and animals [21]. However, considerable differences in leptin gene expression between various fat depots have been observed. For example, leptin mRNA levels were up to 5 times higher in subcutaneous fat than visceral fat in both lean and obese humans [22]. In rodents, leptin mRNA levels were significantly higher in retroperitoneal than in inguinal adipose tissues [23]. The present study adds to the growing body of knowledge on the associations between leptin and ectopic fat depots by showing that, for every $1 \mathrm{ng} / \mathrm{mL}$ change in levels of leptin, there is a $0.04 \%$ increase in IPFD (after adjustment for age, sex, $\mathrm{V} / \mathrm{S}$ fat volume ratio, glycated hemoglobin, and other covariates). This finding implicates leptin as an important contributor to morphology of both the exocrine and endocrine pancreas, which ultimately leads to altered functions of this organ. Leptin is known to promote the production of type 1 collagen and growth factor $\beta 1$ by activated stellate cells [24]. Hence, it is possible that leptin contributes to reduced regenerative capacity of the pancreas, fibrosis, and fatty replacement of pancreatic acini via pancreatic stellate cells [25]. Leptin may also affect the endocrine pancreas as $\beta$ cells are known to express the leptin receptor [26]. Changes in leptin signaling in $\beta$ cells could potentially lead to hyperinsulinemia, exacerbating the adipogenic effect of insulin and resulting in the accumulation of fat $[27,28]$.

This study had several strengths. First, it was informed by 2 complementary systematic reviews on IPFD by the COSMOS group that enabled benchmarking of available evidence on the subject $[3,14]$. Second, as body mass index is a crude proxy for body fat at an individual level, we used MRI as the modern gold standard for measurement of abdominal fat distribution. Further, visceral and subcutaneous fat depots were reported as volumes, not areas
18

Ann Nutr Metab 2022;78:14-20 DOI: $10.1159 / 000520068$
Nguyen/Singh/Petrov 
(the latter is less time-consuming approach but is also less comprehensive). Third, all MRI measurements were done independently by 2 raters (in a blinded fashion) for the entire cohort and yielded excellent inter-rater reliability (intra-class correlation coefficients $>0.90$ ). Fourth, we investigated all the fat depots on a continuous scale because there is a lack of consensus on cutoff values for these. This approach increased generalizability of the reported findings. Last, several robust statistical models were built to investigate the effect of possible confounders, including but not limited to age, sex, glycated hemoglobin, and abdominal fat distribution. The findings across most of the adjusted models were consistent, reducing the possibility that the associations were observed merely due to chance.

One of the main limitations of the present study was its cross-sectional study design. Therefore, no causal inference can be drawn from the presented findings. While the putative pathways described above may explain how increased leptin and the leptin/ghrelin ratios can lead to excess IPFD, one cannot conclude whether increased leptin and the leptin/ghrelin ratio cause high IPFD or the other way around. Also, given that this was the first study in the literature to investigate the association between IPFD and the leptin/ghrelin ratio, we had no data to inform our power calculation. Hence, the study might have been underpowered, overpowered, or adequately powered. Last, albeit the presented estimates were adjusted for several possible confounders (including but not limited to age, sex, and V/S fat volume ratio), it is conceivable that some important covariates of the studied associations were not investigated.

In conclusion, the leptin/ghrelin ratio in the fasted state was significantly directly associated with IPFD. In considering the components of the ratio individually, circulating levels of leptin (but not ghrelin) were significantly directly associated with IPFD. When the observed findings are externally validated and the mechanistic basis of the link between leptin and IPFD is elucidated, leptin can be considered as a potential therapeutic target with a view to reducing the burden of fatty pancreas disease.

\section{Acknowledgment}

The study was part of the COSMOS program.

\section{Statement of Ethics}

The study was approved by the Southern Health and Disability Ethics Committee (13/STH/182). All participants provided their written informed consent.

\section{Conflict of Interest Statement}

The authors have no conflict of interest.

\section{Funding Sources}

This study was supported by the Royal Society of New Zealand (Rutherford Discovery Fellowship to Professor Max Petrov).

\section{Author Contributions}

M.S.P conceived the study. R.G.S. and N.N.N performed patient recruitment. R.G.S. and N.N.N were involved in data acquisition. N.N.N performed analysis and interpretation of data, statistical analysis, and drafting of the manuscript. R.G.S. and M.S.P revised the manuscript. M.S.P supervised the study.

\section{Data Availability Statement}

All data generated or analyzed during this study are included in this manuscript.

\section{References}

1 Wang CY, Ou HY, Chen MF, Chang TC, Chang CJ. Enigmatic ectopic fat: prevalence of nonalcoholic fatty pancreas disease and its associated factors in a Chinese population. J Am Heart Assoc. 2014;3:e000297.

2 Wong VW, Wong GL, Yeung DK, Abrigo JM, Kong AP, Chan RS, et al. Fatty pancreas, insulin resistance, and $\beta$-cell function: a population study using fat-water magnetic resonance imaging. Am J Gastroenterol. 2014;109:58997. http://dx.doi.org/10.1038/ajg.2014.1.
3 Singh RG, Yoon HD, Wu LM, Lu J, Plank LD, Petrov MS. Ectopic fat accumulation in the pancreas and its clinical relevance: a systematic review, meta-analysis, and meta-regression. Metabolism. 2017;69:1-13.

4 Petrov MS. Post-pancreatitis diabetes mellitus and excess intra-pancreatic fat deposition as harbingers of pancreatic cancer. World J Gastroenterol. 2021;27:1936-42.
5 Sreedhar UL, DeSouza SV, Park B, Petrov MS. A systematic review of intra-pancreatic fat deposition and pancreatic carcinogenesis. J Gastrointest Surg. 2020;24:2560-9.

6 Singh RG, Cervantes A, Kim JU, Nguyen NN, DeSouza SV, Dokpuang D, et al. Intrapancreatic fat deposition and visceral fat volume are associated with the presence of diabetes after acute pancreatitis. Am J Physiol Gastrointest Liver Physiol. 2019;316:G806-15. 
7 Quiclet C, Dittberner N, Gässler A, Stadion $\mathrm{M}$, Gerst F, Helms A, et al. Pancreatic adipocytes mediate hypersecretion of insulin in diabetes-susceptible mice. Metabolism. 2019; 97:9-17.

8 Lim EL, Hollingsworth KG, Aribisala BS, Chen MJ, Mathers JC, Taylor R. Reversal of type 2 diabetes: normalisation of beta cell function in association with decreased pancreas and liver triacylglycerol. Diabetologia. 2011;54:2506-14.

9 Al-Mrabeh A, Hollingsworth KG, Shaw JAM, McConnachie A, Sattar N, Lean MEJ, et al. 2 -year remission of type 2 diabetes and pancreas morphology: a post-hoc analysis of the DiRECT open-label, cluster-randomised trial. Lancet Diabetes Endocrinol. 2020;8:93948.

10 Gepner Y, Shelef I, Schwarzfuchs D, Zelicha $\mathrm{H}$, Tene L, Yaskolka Meir A, et al. Effect of distinct lifestyle interventions on mobilization of fat storage pools: CENTRAL magnetic resonance imaging randomized controlled trial. Circulation. 2018;137:1143-57.

11 Friedman JM. Leptin and the endocrine control of energy balance. Nat Metab. 2019;1: 754-64.

12 Camilleri M, Papathanasopoulos A, Odunsi ST. Actions and therapeutic pathways of ghrelin for gastrointestinal disorders. Nat Rev Gastroenterol Hepatol. 2009;6:343-52.

13 Konturek PC, Konturek JW, CześnikiewiczGuzik M, Brzozowski T, Sito E, Konturek SJ. Neuro-hormonal control of food intake: basic mechanisms and clinical implications. J Physiol Pharmacol. 2005;56:5-25.
14 Singh RG, Yoon HD, Poppitt SD, Plank LD, Petrov MS. Ectopic fat accumulation in the pancreas and its biomarkers: a systematic review and meta-analysis. Diabetes Metab Res Rev. 2017;33:e2918.

15 Singh RG, Nguyen NN, DeSouza SV, Pendharkar SA, Petrov MS. Comprehensive analysis of body composition and insulin traits associated with intra-pancreatic fat deposition in healthy individuals and people with newonset prediabetes/diabetes after acute pancreatitis. Diabetes Obes Metab. 2019;21:417-23.

16 Singh RG, Nguyen NN, Cervantes A, Kim JU, Stuart CE, Petrov MS. Circulating levels of lipocalin-2 are associated with fatty pancreas but not fatty liver. Peptides. 2019;119:170117.

17 Singh RG, Nguyen NN, Cervantes A, Cho J, Petrov MS. Serum lipid profile as a biomarker of intra-pancreatic fat deposition: a nested cross-sectional study. Nutr Metab Cardiovasc Dis. 2019;29:956-64.

18 Cervantes A, Singh RG, Kim JU, DeSouza SV, Petrov MS. Relationship of anthropometric indices to abdominal body composition: a multiethnic New Zealand magnetic resonance imaging study. J Clin Med Res. 2019;11:435-46.

19 Modesto AE, Stuart CE, Cho J, Ko J, Singh RG, Petrov MS. Psoas muscle size as a magnetic resonance imaging biomarker of progression of pancreatitis. Eur Radiol. 2020;30: 2902-11.

20 Bharmal SH, Cho J, Stuart CE, Alarcon Ramos GC, Ko J, Petrov MS. Oxyntomodulin may distinguish new-onset diabetes after acute pancreatitis from type 2 diabetes. Clin Transl Gastroenterol. 2020;11:e00132.
21 Maffei M, Halaas J, Ravussin E, Pratley RE, Lee GH, Zhang Y, et al. Leptin levels in human and rodent: measurement of plasma leptin and ob RNA in obese and weight-reduced subjects. Nat Med. 1995;1:1155-61. http:// dx.doi.org/10.1038/nm1195-1155.

22 Masuzaki H, Ogawa Y, Isse N, Satoh N, Okazaki T, Shigemoto M, et al. Human obese gene expression. Adipocyte-specific expression and regional differences in the adipose tissue. Diabetes. 1995;44:855-8.

23 Zhang Y, Guo KY, Diaz PA, Heo M, Leibel RL. Determinants of leptin gene expression in fat depots of lean mice. Am J Physiol Regul Integr Comp Physiol. 2002;282:R226-34.

24 Agrawal S, Gollapudi S, Su H, Gupta S. Leptin activates human B cells to secrete TNF- $\alpha$, IL6, and IL-10 via JAK2/STAT3 and p38MAPK/ ERK1/2 signaling pathway. J Clin Immunol. 2011;31:472-8.

25 Ko J, Stuart CE, Modesto AE, Cho J, Bharmal $\mathrm{SH}$, Petrov MS. Chronic pancreatitis is characterized by elevated circulating periostin levels related to intra-pancreatic fat deposition. J Clin Med Res. 2020;12:568-78.

26 Covey SD, Wideman RD, McDonald C, Unniappan S, Huynh F, Asadi A, et al. The pancreatic beta cell is a key site for mediating the effects of leptin on glucose homeostasis. Cell Metab. 2006;4:291-302.

27 Morioka T, Asilmaz E, Hu J, Dishinger JF, Kurpad AJ, Elias CF, et al. Disruption of leptin receptor expression in the pancreas directly affects beta cell growth and function in mice. J Clin Invest. 2007;117:2860-8.

28 Kieffer TJ, Habener JF. The adipoinsular axis: effects of leptin on pancreatic beta-cells. Am J Physiol Endocrinol Metab. 2000;278:E1-E14. 